\title{
Der Arzt als Diener vierer Herren*
}

Walter Grete

Dr. med., Präsident IG Seniorenärzte

In Goldonis Lustspiel führte bereits die Bedienung zweier Herrschaften zu kaum entwirrbaren Komplikationen. Dem Arzt, der Ärztin der Gegenwart wird ein Spagat zwischen vier fordernden Auftraggebern zugemutet. Ursprünglich diente der Arzt allein dem hilfesuchenden Menschen, dem Patienten. Über Jahrhunderte waren die Heilung, die Linderung und die Begleitung des leidenden Auftraggebers Ziel und Zweck der ärztlichen Tätigkeit.

Im 19. Jahrhundert wurde die Ärzteschaft mehr und mehr der universitären Wissenschaft verpflichtet. Ihre Tätigkeit forderte zunehmend eine Übereinstimmung mit den naturwissenschaftlichen Erkenntnissen. Damit war das Fundament zur sogenannten "Schulmedizin» gelegt. Medizin ohne den zweiten Auftraggeber, «Die Wissenschaft», wurde unvorstellbar. Die grossartigen Fortschritte der Medizin bestätigen, dass der erste Spagat der Ärzteschaft hervorragend gut gelungen ist.

Mit Beginn des 20. Jahrhunderts kam ein dritter, bedeutender Herr in den ärztlichen Wirkungskreis: der Versicherer und mit ihm die Ökonomie des zahlenden Kollektivs. Die karitative Komponente verschwand rasch aus der Krankenversorgung. Die organisierten Prämienzahler forderten Rechenschaft über ihre Beiträge, bis hinunter in die individuelle Tätigkeit des einzelnen Arztes. Die «Bedienung» dieses dritten Herrn ist der Ärzteschaft schwergefallen und fällt ihr noch immer schwer. Die zeitintensiven Rechenschaftsberichte und Formulare an die Versicherungen dienen der Dokumentation, der Leistungsberechtigung, aber auch den Begriffen Wirksamkeit, Zweckmässigkeit und Wirtschaftlichkeit (WZW). Diese Fragestellung, ob die geleistete ärztliche Hilfe im Einzelfall den sogenannten WZW-Kriterien entsprochen hat, kann bei allem Aufwand meist erst sehr viel später beantwortet werden.

Der dritte Spagat ist der Ärzteschaft somit trotz immensem Zeitaufwand nur teilweise gelungen. Er hat aber zusätzlich die Versicherungsökonomie und mit ihr Misstrauen und eine neue Partei in die Arzt-Patienten-Beziehung gebracht. Die Dreiecksbeziehung ArztPatient-Versicherung wurde strapaziert und auch kompliziert. Den Ausweg aus dieser latenten Beziehungskriese sehen Gesundheitspolitiker, Versicherungsfachleute und natürlich die Ökonomen in der Verbesserung der «Zahlen-Grundlagen» zur Lenkung und Überwachung der ärztlichen Tätigkeiten und Leistungen.
An dieser Stelle gesellt sich nun der vierte Herr zum dienenden Arzt: Die «Digitalisierung» des ärztlichen Tagewerks. Eine breite Palette von Kontrollstatistiken wurde aufgebaut, die alle bedient sein wollen: Seien es medizinische Statistiken über Fallzahlen, über die ärztlichen Tagesstrukturen, die Minuten am Patienten und dessen Zeitverlust im Wartezimmer, über den Umfang und die Haftpflichtqualität von Arztberichten, über Temperaturtabellen im ärztlichen Kühlschrank, die Statistiken zu Todesursachen, über Nebenwirkungen von Medikamenten, ein detailliertes Krebsregister [1], die Erfassung ansteckender Krankheiten, die Meldungen über Fortbildungsstunden im Hörsaal und am Schreibtisch, aber auch Meldungen zu Beinahe-Fehlern, zu Grippe, zu Suchtpatienten und ... und - und nicht zuletzt ein Krämertarif mit einer Detailtiefe, die ihn unlesbar macht. Zahlen über Zahlen. "Big Data», eine unendliche Liste. Und wer füttert diese tagtäglich mit all den Zahlen? Die geplagte Ärztin und der ge-

\section{Niemand kann mehreren Herren uneingeschränkt dienen.}

plagte Arzt, Magd und Knecht ihrer vier Herren. Weshalb diese Geschichte? Niemand kann mehreren Herren uneingeschränkt dienen. In der geschilderten Entwicklung sind es die Patientinnen und Patienten, die zu kurz kommen. Der Versuch, die Ökonomie des Gesundheitswesens über Zahlen und Statistiken in den Griff zu bekommen, kann nur durch eine rigorose Entlastung der Ärzteschaft von Aufgaben der Administration und der «Fütterung» von Computern gelingen. Die absorbierten Ärzte fehlen am Krankenbett, wenn sie zeitfressend für die Erfüllung berufsfremder Aufgaben missbraucht werden. Ihre Aus-, Weiter- und Fortbildungszeiten verlängern sich überproportional. Die selbst beurteilten Fallzahlen des einzelnen Arztes sinken, sein Erfahrungsschatz leidet. Es wäre zu prüfen, inwieweit die unzähligen Dauerdaten-Erhebungen mit ihrem gewaltigen Apparat mehr und mehr zu Mitgestaltern eines ineffizienten Gesundheitswesens werden. Mit einer neuen Statistik liesse sich das Ausmass des bürokratischen Aufwandes wohl beweisen ...

\section{Literatur}

1 Schuurmans M. M. Müssen wir uns diesen bürokratischen Wahnsinn gefallen lassen? Schweiz Ärzteztg. 2019;100(49):1677-8. 